OPEN ACCESS

Edited by:

George Tsiamis,

University of Patras, Greece

Reviewed by:

Pieter Arnold,

Australian National University,

Australia

Ewa Anna Chrostek,

University of Liverpool,

United Kingdom

*Correspondence:

Xiao-Yue Hong

xyhong@njau.edu.cn

Specialty section:

This article was submitted to

Microbial Symbioses,

a section of the journal

Frontiers in Microbiology

Received: 07 May 2021

Accepted: 14 June 2021

Published: 07 July 2021

Citation:

Zhu Y-X, Song Z-R, Zhang $Y$-Y, Hoffmann AA and Hong $X-Y$ (2021) Spider Mites Singly Infected With Either Wolbachia or Spiroplasma Have Reduced Thermal Tolerance.

Front. Microbiol. 12:706321. doi: 10.3389/fmicb.2021.706321

\section{Spider Mites Singly Infected With Either Wolbachia or Spiroplasma Have Reduced Thermal Tolerance}

\author{
Yu-Xi Zhu',2, Zhang-Rong Song', Yi-Yin Zhang ${ }^{1}$, Ary A. Hoffmann ${ }^{3}$ and Xiao-Yue Hong ${ }^{1 *}$ \\ ${ }^{1}$ Department of Entomology, Nanjing Agricultural University, Nanjing, China, ${ }^{2}$ Institute of Applied Entomology, School \\ of Horticulture and Plant Protection, Yangzhou University, Yangzhou, China, ${ }^{3}$ School of BioSciences, Bio21 Institute, \\ The University of Melbourne, Melbourne, VIC, Australia
}

Heritable symbionts play an essential role in many aspects of host ecology in a temperature-dependent manner. However, how temperature impacts the host and their interaction with endosymbionts remains largely unknown. Here, we investigated the impact of moderate $\left(20^{\circ} \mathrm{C}\right)$ and high $\left(30\right.$ and $\left.35^{\circ} \mathrm{C}\right)$ temperatures on symbioses between the spider mite Tetranychus truncatus and two maternally inherited endosymbionts (Wolbachia and Spiroplasma). We found that the thermal tolerance of mites (as measured by survival after heat exposure) was lower for mites that were singly infected with either Wolbachia or Spiroplasma than it was for co-infected or uninfected mites. Although a relatively high temperature $\left(30^{\circ} \mathrm{C}\right)$ is thought to promote bacterial replication, rearing at high temperature $\left(35^{\circ} \mathrm{C}\right)$ resulted in losses of Wolbachia and particularly Spiroplasma. Exposing the mites to $20^{\circ} \mathrm{C}$ reduced the density and transmission of Spiroplasma but not Wolbachia. The four spider mite strains tested differed in the numbers of heat shock genes (Hsps) induced under moderate or high temperature exposure. In thermal preference (Tp) assays, the two Wolbachia-infected spider mite strains preferred a lower temperature than strains without Wolbachia. Our results show that endosymbiont-mediated spider mite responses to temperature stress are complex, involving a combination of changing endosymbiont infection patterns, altered thermoregulatory behavior, and transcription responses.

Keywords: Wolbachia, Spiroplasma, Tetranychus truncatus, thermal tolerance, thermal preference

\section{INTRODUCTION}

Temperature has a substantial impact on a wide range of ecologically important traits in many ectothermic organisms (Colinet et al., 2015; Sgro et al., 2016; Ma et al., 2021) and their symbiotic microbiota (Kiers et al., 2010). Heritable bacterial symbionts, which are pervasive in many arthropods, affect the host's response to abiotic stressors, including temperature (Lemoine et al., 2020). Bacterial endosymbionts can both mediate and constrain host adaptation to temperature 
extremes (Corbin et al., 2017). For example, the obligate bacterial endosymbiont, Buchnera, limits the thermal tolerance of its aphid hosts and thus represents an "Achilles' heel" of their thermal response (Dunbar et al., 2007; Zhang et al., 2019), while the facultative endosymbiont Serratia symbiotica increases aphid survival or reproduction under heat exposure likely through releasing metabolites (Montllor et al., 2002; Burke et al., 2010). Some strains of Wolbachia can increase Drosophila melanogaster's thermotolerance by accelerating dopamine metabolism (Gruntenko et al., 2017), while Hamiltonella infections in whitefly also confer a fitness advantage under heat that may involve induction of the expression of host-produced stress genes (Brumin et al., 2011). Thermal effects mediated by symbionts may therefore affect host distribution as well as responses to ongoing changing climate.

Conversely, the persistence and stability of the bacterial symbionts themselves can be affected by temperature stress (Wernegreen, 2012), which can impact facultative endosymbiont dynamics through influencing their density, transmission, and phenotypic effects (Renoz et al., 2019). For instance, heat stress from exposure to high temperatures $\left(>30^{\circ} \mathrm{C}\right)$ reduces Wolbachia density in various species, including Aedes aegypti (Ross et al., 2020), D. simulans (Hoffmann et al., 1986), and Nasonia vitripennis (Bordenstein and Bordenstein, 2011), while in aphids it affects densities of the nutritional obligate symbiont Buchnera (Zhang et al., 2019). Reduced Wolbachia density results in weaker cytoplasmic incompatibility (CI) which assists endosymbionts like Wolbachia to spread in populations, and reduced endosymbiont density can also lead to maternal transmission failure (Ross et al., 2017). Other host species such as Acyrthosiphon pisum, that have a complex mixture of symbionts, can show a recovery of their obligate symbionts after heat stress that depends on their facultative symbionts (Heyworth et al., 2020). Thus, impacts of thermal conditions on symbionts may vary depending on the nature of the stress, host species, and symbiont community.

The spider mite, Tetranychus truncatus, is a dominant mite species in China (Jin et al., 2018) and is frequently associated with multiple endosymbionts including Wolbachia and Spiroplasma (Zhu et al., 2018). Both these symbionts are maternally inherited and can co-infect the same $T$. truncatus host. Wolbachia induces incomplete CI in spider mites (Yang et al., 2020). A comprehensive survey of endosymbionts in the natural population of $T$. truncatus has shown that the incidence of endosymbionts is associated with environmental factors. In particular, Wolbachia infection rates increase at localities with higher annual mean temperatures (Zhu et al., 2018). This is in contrary to high temperatures negatively impacting Wolbachia transmission and CI strength in the related mite T. urticae (van Opijnen and Breeuwer, 1999), which may be correlated with the lytic activity of phage WO (Lu et al., 2012). Wolbachia-induced CI phenotype and transmission are therefore temperature dependent in spider mites; however, the impact of temperature on endosymbiont interactions is not clear in T. truncatus.

In the present study, we investigated the impact of high and low temperatures on the survival of females from $T$. truncatus strains that were either uninfected or singly and doubly infected with Wolbachia and Spiroplasma strains. We also tested the effect of temperature stress on endosymbiont density and vertical transmission efficiency, and we explored the transcriptome to investigate possible mechanisms associated with any endosymbiont-mediated temperature responses. Finally, we tested whether infection with Wolbachia and Spiroplasma affected host thermal preference (Tp). We hypothesized that spider mites infected with different symbiont strains would display differences in thermal tolerance and Tp; in turn, symbioses of Wolbachia and Spiroplasma with T. truncatus would be affected by temperature stress.

\section{MATERIALS AND METHODS}

\section{Spider Mite Strains and Rearing}

Four spider mite strains with a similar genetic background were established (Yang et al., 2020; Zhu et al., 2020): strains co-infected with Wolbachia and Spiroplasma (designated as $w+s+)$, Wolbachia only $(w+)$, Spiroplasma only $(s+)$, or no symbionts $(w-s-)$. Symbiont-infected mite strains $(w+s+$, $w+$, and $s+$ ) were collected from Shenyang, Liaoning Province, China. A $w$-s- strain was obtained by tetracycline treatment of the $s+$ strains for three generations. To eliminate potential effects of the tetracycline, we cultivated the $w$-sstrain without tetracycline treatment for 15 generations. To minimize genetic background differences between four spider mite strains, we mated infected female adults with the uninfected male adults and backcrossed female offspring for at least seven consecutive generations.

Spider mite strains were reared on leaves of the common bean (Phaseolus vulgaris L.) placed on a water-saturated sponge mat in a Petri dish at $25 \pm 1{ }^{\circ} \mathrm{C}$ and $60 \%$ relative humidity and under $16 \mathrm{~h}$ light: $8 \mathrm{~h}$ dark conditions. Prior to the experiment, the infection status was checked as by PCR (Yang et al., 2020). A schematic of the study design is given in Supplementary Figure 1.

\section{Survival at Constant Temperatures}

To determine survival of the four strains following different temperature treatment, we exposed 20 female adults (2-day) to either $20,25,30$, or $35^{\circ} \mathrm{C}$ in an incubator and then monitored spider mite survival daily for a week using a stereo microscope. Six biological replicates for each treatment were tested.

\section{Crossing Experiments}

Wolbachia-induced incomplete CI was determined through crosses as previously described (Yang et al., 2020). To test the impact of different temperatures on the strength of CI, females without Wolbachia (o: $s+$ or $w$-s-) were crossed with males infected with Wolbachia $\left(\sigma^{\top}: w+s+\right.$ or $\left.w+\right)$, producing a total of four crosses. Single females in the teleiochrysalis stage and a 1day-old adult virgin male were placed on the same leaf disk, which was subjected to one of four temperature treatments: 20, 25, 30, or $35^{\circ} \mathrm{C}$. Each cross and treatment combination was replicated $18-$ 99 times using different leaf disks. Females were allowed to lay 
eggs for 5 days while maintaining treatment temperatures, and then removed. Eggs on leaf disks were continued to be kept under their respective treatment temperatures and were checked daily to calculate hatchability.

\section{Transmission Efficiency of Wolbachia and Spiroplasma}

To examine the vertical transmission of Wolbachia and Spiroplasma, spider mites from each strain were placed on bean leaf discs (diameter ca. $3 \mathrm{~cm}$ ) and were reared for four generations under $20,25,30$, or $35^{\circ} \mathrm{C}$. Twenty female adults ( 2 days old) were randomly selected from each treatment and generational combination (F1 to F4) of these cultures and used in PCR analyses. Six biological replicates were set up per strain. DNA was extracted from a single mite using a DNeasy blood and tissue kit (Qiagen, Hilden, Germany) according to the manufacturer's protocols. Wolbachia and Spiroplasma infections were screened by PCR amplification using the specific primers listed in Supplementary Table 1. PCRs were carried out using a Veriti thermocycler (ABI Biosystems, United States) in a $25 \mu \mathrm{l}$ volume containing $12.5 \mu \mathrm{l} 2 \times$ rapid Taq master mix (Vazyme Biotech, China), $0.5 \mu \mathrm{l}$ primers (20 $\mu \mathrm{M}$ each), and $1 \mu \mathrm{l}$ of DNA extract. PCR cycling parameters were $95^{\circ} \mathrm{C}$ for $3 \mathrm{~min}$, followed by 35 cycles of $95^{\circ} \mathrm{C}$ for $15 \mathrm{~s}$, the annealing temperature for $45 \mathrm{~s}$, and $72^{\circ} \mathrm{C}$ for $15 \mathrm{~s}$, and then $72^{\circ} \mathrm{C}$ for $5 \mathrm{~min}$ at the end. PCRs included a positive and negative control and were run on a $1 \%$ agarose gel with ethidium bromide to visualize the product.

\section{Densities of Wolbachia and Spiroplasma}

To estimate the dynamics of Wolbachia and Spiroplasma following different temperature exposures, the ratio of the singlecopy genes $16 S$ rRNA (Spiroplasma) and wsp (Wolbachia) to the rps-18 (spider mite host) reference gene was determined by realtime qPCR (Yang et al., 2020). Spider mites subjected to the four different temperatures were sampled at 6,12 , and $24 \mathrm{~h}$, as well as on the third and fifth days. For each time point, nine biological replicates were tested per treatment. For each of the biological replicates, we performed three technical replicates. Densities of Wolbachia and Spiroplasma were estimated by qPCR with the ABI QuantStudio 6 Flex (Applied Biosystems, CA, United States). The $20-\mu$ l Q-PCR reaction mixture consisted of $10 \mu 12 \times$ SYBRP remix Ex Taq (Vazyme, China), $0.4 \mu \mathrm{l} 10 \mathrm{mmol} / \mathrm{L}$ of each primer, $0.4 \mu \mathrm{l} 50 \times$ ROX Reference Dye, $2 \mu \mathrm{l}$ DNA template, and $6.8 \mu \mathrm{l} \mathrm{H} \mathrm{H}_{2} \mathrm{O}$ in single wells of a 96-well plate (PE Applied Biosystems, CA, United States). The Q-PCR cycling conditions included one cycle $\left(5 \mathrm{~min}\right.$ at $\left.95^{\circ} \mathrm{C}\right)$ followed by 40 cycles $(10 \mathrm{~s}$ at $95^{\circ} \mathrm{C}$ and $34 \mathrm{~s}$ at $60^{\circ} \mathrm{C}$ ), and finally one cycle to produce melting curves $\left(15 \mathrm{~s}\right.$ at $95^{\circ} \mathrm{C}, 1 \mathrm{~min}$ at $60^{\circ} \mathrm{C}$, and $15 \mathrm{~s}$ at $\left.95^{\circ} \mathrm{C}\right)$. The primers are listed in Supplementary Table 1. Standard curves were plotted using a 10-fold dilution series of the DNA samples prepared from plasmid DNA. The plasmid DNA was obtained with the pEASY-T3 vector (TransGen Biotech, Beijing, China). The quality and concentration of all purified standard DNA were measured on a Nanodrop 2000 (Thermo Scientific, MA, United States).

\section{Transcriptome Processing and Annotation}

To explore transcriptional responses to short-term high or low temperature exposure in the four spider mite strains, we sampled 2 -day-old female adults with exposure to either 20, 25, 30, or $35^{\circ} \mathrm{C}$ for $6 \mathrm{~h}$. About 100 adult female spider mites (2-dayold) were collected for each replicate and then frozen in liquid nitrogen prior to RNA extraction. Four biological replicates for each treatment were tested.

Total RNA was extracted from each sample using Trizol protocol according to manufacturer's instructions (Invitrogen, CA, United States). RNA quality was qualified and quantified using a Nano Drop and Agilent 2100 bioanalyzer (Thermo Fisher Scientific, MA, United States). Construction of cDNA libraries and subsequent sequencing using the BGIseq500 platform were conducted at BGI-Shenzhen, China. Raw data were filtered using SOAPnuke v1.5.2 by removing reads containing adapters, poly-N, and low-quality reads to obtain clean data. Different gene expression was analyzed using the DESEQ2 package (Love et al., 2014) with $Q$ value $\leq 0.05$. Kyoto Encyclopedia of Genes and Genomes (KEGG) enrichment analysis and Gene Ontology (GO) were based on the KEGG pathway database ${ }^{1}$ and the GO database ${ }^{2}$.

To confirm the results of the RNA-seq analysis, the expression levels of randomly selected genes were determined by RT-qPCR. The RT-qPCR reactions were performed on an ABI QuantStudio 6 Flex Real-Time PCR System with SYBR Premix Ex Taq (Takara Bio, Kyoto, Japan). The amplification reactions were performed in a $20 \mu \mathrm{L}$ final volume containing $10 \mu \mathrm{L}$ of TaKaRa buffer, $7.2 \mu \mathrm{L}$ of $\mathrm{ddH}_{2} \mathrm{O}_{2}, 0.4 \mu \mathrm{L}$ of DyeII (TaKaRa), $0.2 \mu \mathrm{L}$ of forward primer $(5 \mathrm{mM})$ and reverse primer $(5 \mathrm{mM})$, and $2 \mu \mathrm{L}$ of cDNA first-strand template. Thermal cycling conditions were as follows: $95^{\circ} \mathrm{C}$ for $10 \mathrm{~min}$, then 40 cycles of $5 \mathrm{~s}$ at $95^{\circ} \mathrm{C}$, and $34 \mathrm{~s}$ at $60^{\circ} \mathrm{C}$. There were three technical replicates for each sample. Primer sequences were designed using Primer Premier 6.0, and are listed in Supplementary Table 1. Expression levels for each gene were calculated by the $2^{-\Delta \Delta C T}$ method (Livak and Schmittgen, 2001).

\section{Thermal Preference Assays}

Temperature preference assays were performed using a custombuilt thermal gradient apparatus consisting of a 500-mm-long aluminum bar along which a temperature gradient $\left(10-40^{\circ} \mathrm{C}\right)$ created with water baths at the ends. Six grooves were etched along the aluminum bar which allowed mites to move freely up or down the gradient without interacting with each other (Supplementary Figure 2). All assays were conducted in a room with a constant temperature of $25^{\circ} \mathrm{C}$ and constant $40 \%$ humidity. For each data point, six individuals of the same strain were, respectively, transferred to the center point of each groove in the apparatus $\left(25^{\circ} \mathrm{C}\right)$ and allowed to move freely along its groove for $30 \mathrm{~min}$. The temperatures at the positions of rest for the six spider mites after $30 \mathrm{~min}$, as measured with $\mathrm{K}$-type thermocouples, were used to calculate a mean preferred temperature. In total, more

\footnotetext{
${ }^{1}$ http://www.genome.jp/kegg/

${ }^{2}$ http://www.geneontology.org/
} 


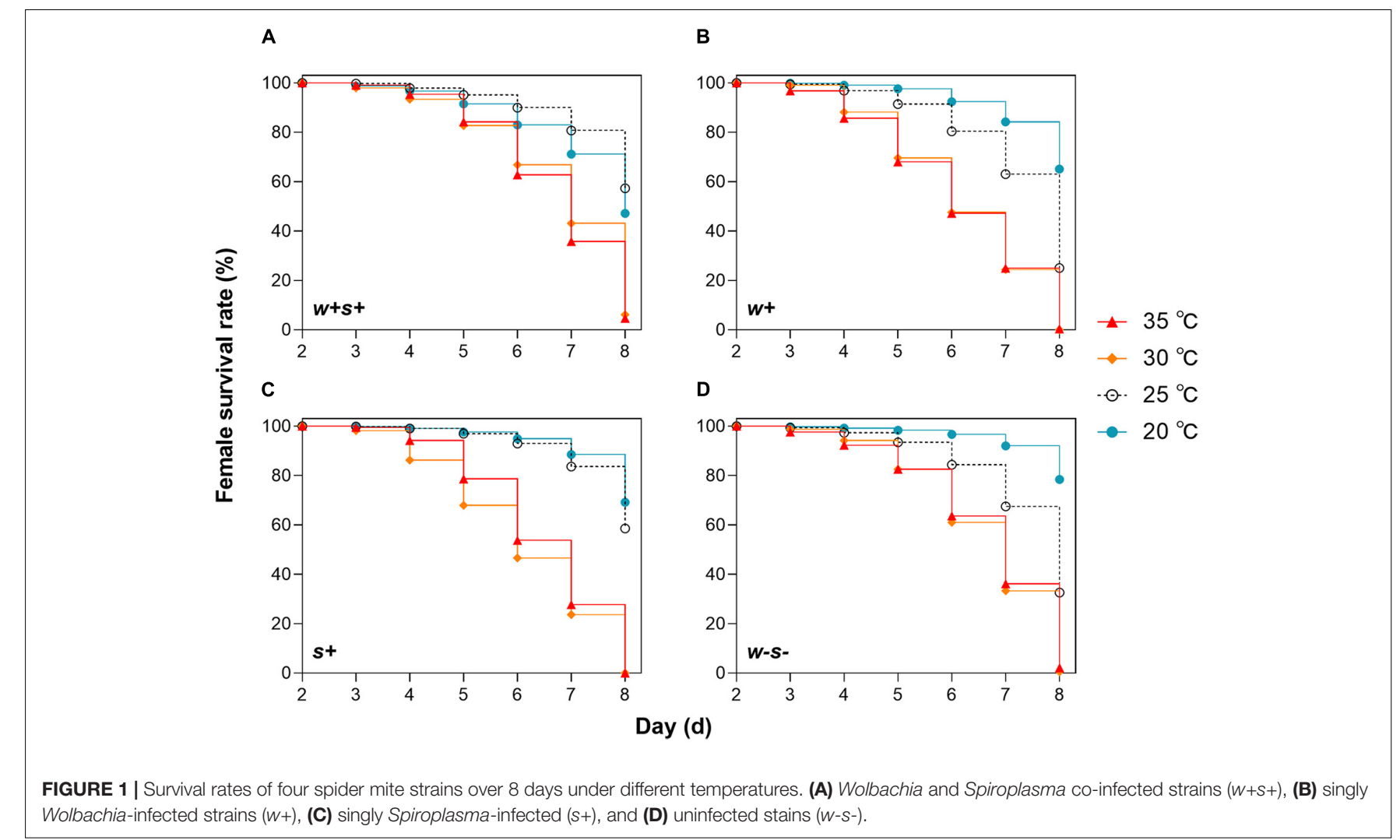

than 282 individuals from each spider mite strain were used to test host Tp.

\section{Statistical Analysis}

All statistical analyses were carried out in $\mathrm{R}$ ver 3.3.1 or GraphPad Prism 9.0 (GraphPad Software Inc., San Diego, CA, United States). Log-rank (Mantel-Cox) tests were used to compare the survival proportions of the spider mite strains under different temperatures. We also compared differences in survival rate between each treatment at the same time point using two-way ANOVAs with multiple comparisons. We used either ANOVAs or Mann-Whitney tests to compare endosymbionts densities, egg hatch proportions, and frequencies of endosymbiont infection, depending on whether data were normally distributed or deviated from normality based on Kolmogorov-Smirnov tests. We conducted a non-parametric Kruskal-Wallis test to compare the Tp values. Differences were considered significant at $p<0.05$ for all analyses.

\section{RESULTS}

\section{Singly Infected Wolbachia or Spiroplasma Strains Have Lower Fitness Than a Co-infected Strain at Higher Temperatures}

We first compared female survival for mites reared under either $20,25,30$, or $35^{\circ} \mathrm{C}$. The survival rate of four spider mite strains was significantly $(p<0.0001)$ affected by temperature (log-rank test, $w+s+: \chi^{2}=311.2, d f=3 ; w+$ : $\chi^{2}=660.8, d f=3 ; s+: \chi^{2}=885.0, d f=3 ; w-s-: \chi^{2}=522.5$, $d f=3$; Figure 1). After 2-4 days, the female survival rates of co-infected strains were not significantly different at 25, 30 , and $35^{\circ} \mathrm{C}$, but after 4 days, the female survival rate of the other three strains was significantly lower at the higher temperatures $\left(30\right.$ and $35^{\circ} \mathrm{C}$ ) than at $25^{\circ} \mathrm{C}$ (Figure 1). This suggests that co-infection with Wolbachia and Spiroplasma delayed high temperature effects on the host. Moreover, when the four mite strains were reared at 30 and $35^{\circ} \mathrm{C}$, the female survival rate after 5 days was sharply lower in the singly infected strains than in the co-infected and uninfected strains (Figure 1). These results indicate that spider mites singly infected with Wolbachia or Spiroplasma are sensitive to high temperatures. In contrast, female survival rate did not differ significantly between 20 and $25^{\circ} \mathrm{C}$ for either co-infected or Spiroplasma-infected strains at any time point. However, survival did sharply decrease at $25^{\circ} \mathrm{C}$ in Wolbachia-infected and uninfected strains after 5 days (Figure 1). Spider mites infected with Spiroplasma may therefore have a higher fitness at the intermediate temperature.

\section{Higher and Lower Temperatures Modify the Cl Strength of Wolbachia}

Consistent with previous results (Yang et al., 2020), we found that Wolbachia induced incomplete CI, resulting in 20-30\% offspring mortality when mites were reared at $25^{\circ} \mathrm{C}$. Embryos 
showed aborted development when CI occurred (Figure 2A). Exposure to a high temperature $\left(35^{\circ} \mathrm{C}\right)$ resulted in a significant decrease in the mean egg hatch rate in the cross between Spiroplasma-infected females and co-infected or Wolbachiainfected mites compared to those same crosses at $25^{\circ} \mathrm{C}$ (MannWhitney test, $w+s+\sigma^{7} \times s+$ o: $35^{\circ} \mathrm{C}$ vs. $25^{\circ} \mathrm{C}$, $p=0.0025 ; w+\sigma^{\top} \times s+\circ: 35$ vs. $\left.25^{\circ} \mathrm{C}, p=0.0002\right)$ (Figure 2B). The hatch proportion significantly declined at the lower temperature in the cross between co-infected males and uninfected females at $20^{\circ} \mathrm{C}$ compared to those at $25^{\circ} \mathrm{C}$, from $77.78 \%$ at $25^{\circ} \mathrm{C}$ to $69.39 \%$ at $20^{\circ} \mathrm{C}$ (Mann-Whitney test: $p=0.0004)$. For the crosses between uninfected females and Wolbachia-infected males, there was no significant difference in the hatch proportion of offspring exposed to either $20,25,30$, or $35^{\circ} \mathrm{C}$ (Figure 2B).

\section{Higher Temperatures Affect Endosymbiont Titer in Female Hosts}

We measured the effect of temperature stress on Wolbachia and Spiroplasma density in female hosts. Wolbachia titers in co-infected strains exposed to $35^{\circ} \mathrm{C}$ were significantly higher than in these strains at $25^{\circ} \mathrm{C}$ at days 1,3 , and 5 , but no significant difference between strains was found at 20 and $25^{\circ} \mathrm{C}$ at all time points (Figure 3A). In contrast to co-infected strains, Wolbachia titers in Wolbachia singly infected strains at either 20 and $35^{\circ} \mathrm{C}$ were significantly lower than those at 30 or $25^{\circ} \mathrm{C}$ at day 3 (Figure 3B). Spiroplasma densities in both co-infected and Spiroplasma-infected strains exposed to either 30 or $35^{\circ} \mathrm{C}$ were significantly higher than those strains at either 20 or $25^{\circ} \mathrm{C}$ at day 0.5 (Figures 3C,D). Spiroplasma densities in co-infected strains fluctuated at later time points under high and low temperatures, while there were no significant differences in Spiroplasma densities in singly infected strains among temperature treatments at days 1, 3, and 5 (Figures 3C,D). The average titer and the shifts in titer with age varied across spider mite strains.

\section{High Temperatures Reduce Infection Frequency of Both Wolbachia and Spiroplasma}

We tested the Wolbachia or Spiroplasma infection frequency in co-infected and singly infected strains over four generations when mites were held at $20,25,30$, or $35^{\circ} \mathrm{C}$. At $25^{\circ} \mathrm{C}$, the normal rearing temperature for host spider mites, both Wolbachia and Spiroplasma were stably maintained with $100 \%$ prevalence in either co-infected or singly infected strains through successive host generations (Figures 4AD). However, Spiroplasma was lost in both co-infected and singly infected strains when mites were reared at $35^{\circ} \mathrm{C}$ for one generation (Figures 4C,D). The prevalence of Wolbachia in singly infected strains sharply decreased from $97.5 \%$ in the F1s to $14.2 \%$ in the F2s and the infection was nearly lost in later generations, but in co-infected mites, the reduction at $35^{\circ} \mathrm{C}$ was less (Figures 4 A,B). At $30^{\circ} \mathrm{C}$, Wolbachia maintained nearly $100 \%$ prevalence in both coinfected and singly infected strains. In contrast, the prevalence

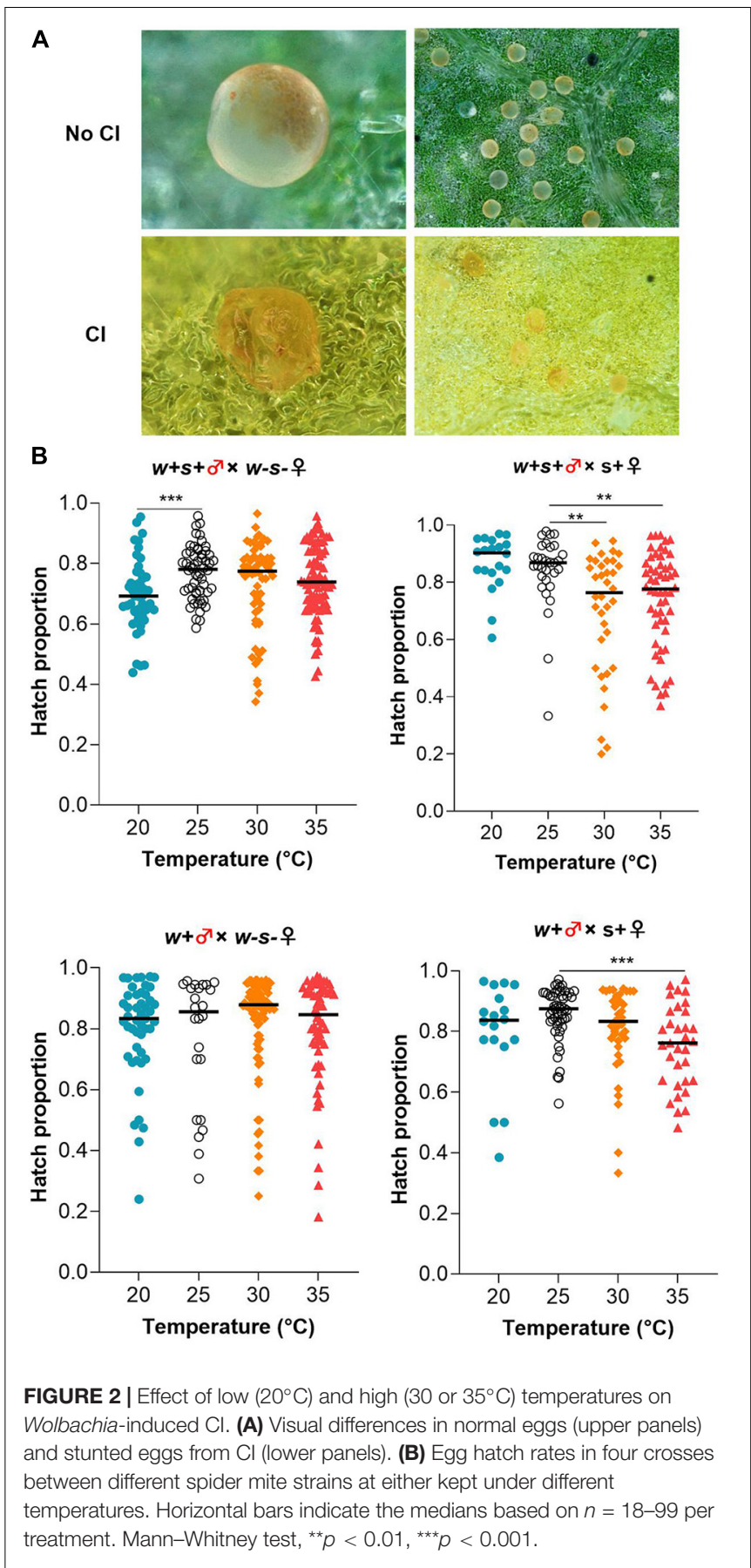

of Spiroplasma in singly infected strains decreased from $90.8 \%$ in the $\mathrm{F} 1 \mathrm{~s}$ to $55.8 \%$ in the $\mathrm{F} 2 \mathrm{~s}$, while the prevalence of Spiroplasma in co-infested strains fluctuated across the four generations (Figures 4C,D). These results suggest that high temperature reduces vertical transmission efficiency of Wolbachia or Spiroplasma in all spider mite strains with varying degrees, and Spiroplasma is more sensitive to high temperatures than Wolbachia. Exposure to a cooler temperature $\left(20^{\circ} \mathrm{C}\right)$ did not affect the infection frequencies of Wolbachia and Spiroplasma in singly infected strains. However, the Spiroplasma 


\section{A}

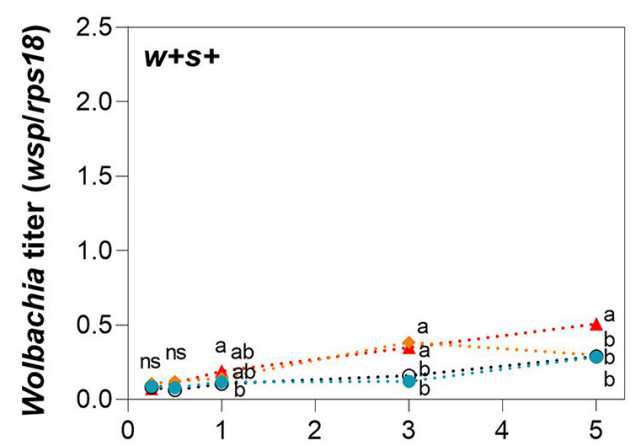

C

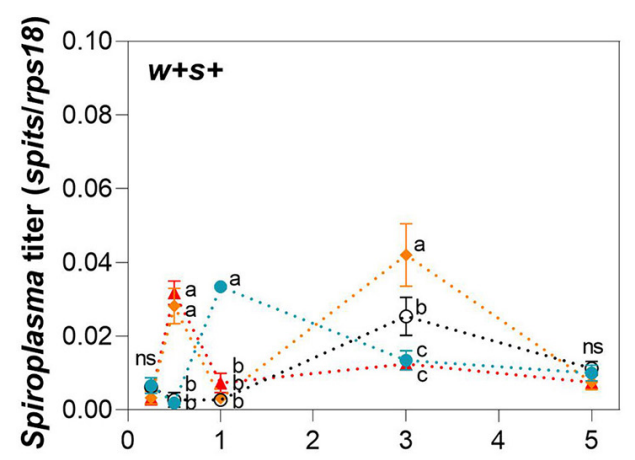

B

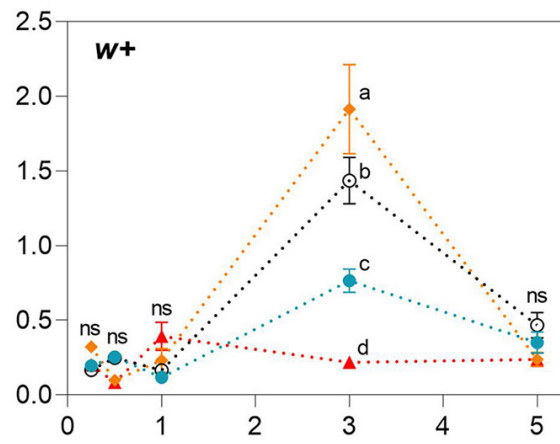

D

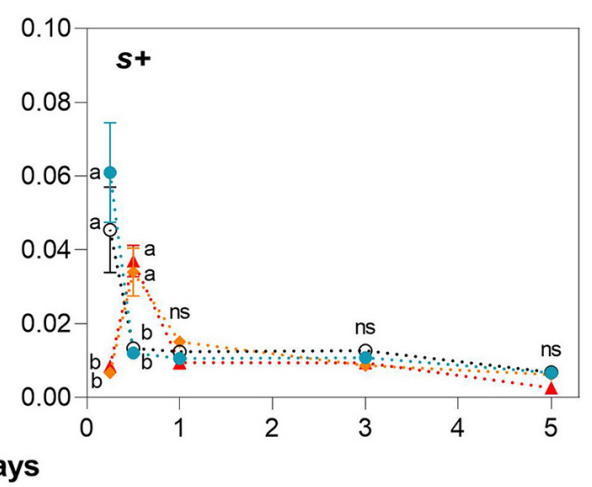

- $20^{\circ} \mathrm{C}$

.๑. $25^{\circ} \mathrm{C}$

-. $30^{\circ} \mathrm{C}$

4. $35^{\circ} \mathrm{C}$

FIGURE 3 | Wolbachia and Spiroplasma densities in female adult spider mites maintained under low (20 $\left.{ }^{\circ} \mathrm{C}\right)$ and high $\left(30\right.$ or $\left.35^{\circ} \mathrm{C}\right)$ temperatures for up to 5 days. Wolbachia density in co-infected strains (A) and Wolbachia-infected strains (B). Spiroplasma density in co-infected strains (C) and Spiroplasma-infected strains (D). Data are shown as the mean \pm SEM. Different letters indicate significant differences between each treatment at the same time point ( $\rho<0.05$; ns, no significant).

infection rate at this temperature in co-infected strains rapidly decreased from $88.3 \%$ in the F1s to a low level of $25 \%$ in the F3s, before increasing in later generations to $80.8 \%$. The Wolbachia infection also varied somewhat in the coinfected strain across generations when mites were held at $20^{\circ} \mathrm{C}$ (Figure 4A).

\section{Gene Expression Differentiation Among Four Spider Mite Strains Under High and Low Temperatures}

Comparison of transcriptional responses to high and low temperatures was based on a total of 2,685.47 $\mathrm{Mb}$ clean reads from 63 libraries derived from four spider mite strains (Supplementary Table 1). Unigenes were annotated to the KEGG and GO databases (Supplementary Figure 3). The PCoA analysis explained $87.88 \%$ of the variance in gene expression of the four spider mite strains under different temperatures (Figure 5A). PCoA demonstrated that the expression patterns of the singly infected Wolbachia strain and singly infected Spiroplasma strain were closely related at all temperatures (Figure 5A).

Variation in gene expression between temperatures was analyzed through comparisons of 35 vs. $25^{\circ} \mathrm{C}, 30$ vs. $25^{\circ} \mathrm{C}$, and 20 vs. $25^{\circ} \mathrm{C}$. The doubly infected strain had the fewest differentially expressed genes compared with the other three mite strains under both high and low temperature exposure (Figure 5B-note scale differences). For the low temperature $\left(20^{\circ} \mathrm{C}\right)$ comparison, Spiroplasma-infected strains showed the most differently expressed genes (DEGs). For $35^{\circ} \mathrm{C}$ comparison, the number of DEGs in un-infected strains was higher than that in the other three spider mite strains when compared to the control treatments.

Among the DEGs, expression of the heat shock protein genes, including Hsp20, Hsp70, and Hsp90, varied in particular between the four spider mite strains. Within the Hsp20 superfamily, a total of 11, two, and six orthologous genes were strikingly up-regulated in Spiroplasma-infected, Wolbachia-infected, and uninfected spider mite strains when spider mites were reared at 35 vs. $25^{\circ} \mathrm{C}$. In contrast, two Hsp genes, Hsp70 and Hsp90, were significantly down-regulated in Spiroplasma-infected strains under low temperature exposure (Figure 5C). We selected eight genes for RT-qPCR validation of differential expression in all samples. The results showed a concordant direction of change between the qPCR and transcriptomic analyses (Supplementary Figure 4).

\section{Effects of Endosymbionts on Thermal Preference in Spider Mite}

We used a custom-built thermal gradient to test whether Wolbachia and Spiroplasma affected temperature preference. 

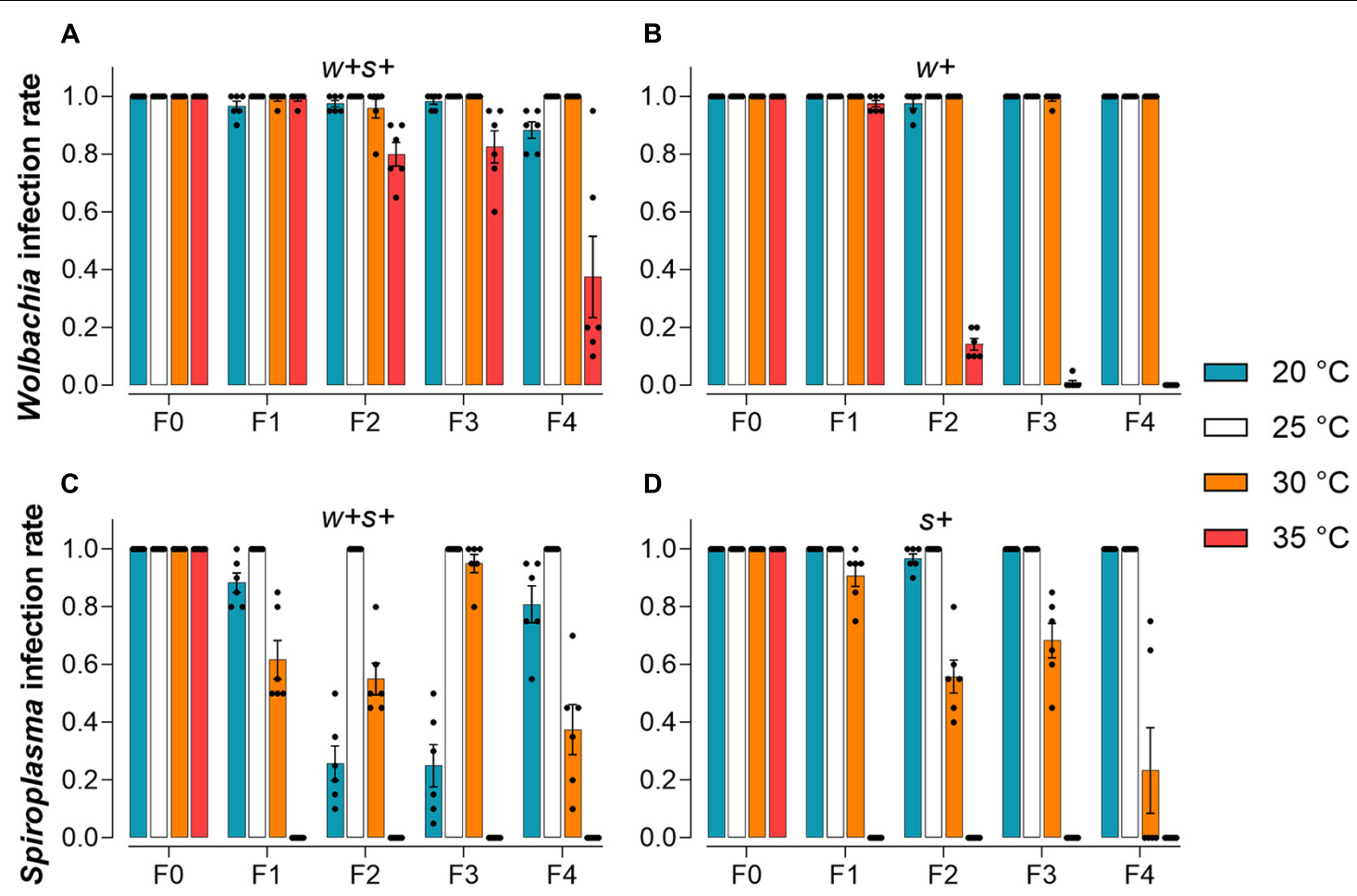

Generation

FIGURE 4 | Infection frequencies of two endosymbionts in female adult spider mites reared for four generations under different temperature conditions. Infection rate of Wolbachia in co-infected strains (A) and Wolbachia-infected strains (B). Infection rate of Spiroplasma in co-infected strains (C) and Spiroplasma-infected strains (D).

The four spider mite strains exhibited a large variance in $\mathrm{Tp}$, falling between 11.80 and $33.40^{\circ} \mathrm{C}$ (Figure $\mathbf{6 A}$ ). There was some overlap in $\mathrm{Tp}$ distributions between the four strains (Figure 6B). However, the preferred average temperature of Wolbachia and Spiroplasma co-infected strains $\left(\mathrm{Tp}=20.74 \pm 0.22^{\circ} \mathrm{C}\right)$ and Wolbachia-infected strains $\left(\mathrm{Tp}=20.21 \pm 0.20^{\circ} \mathrm{C}\right)$ were significantly lower than either Spiroplasma-infected $\left(\mathrm{Tp}=22.31 \pm 0.20^{\circ} \mathrm{C}\right)$ or uninfected strains $\left(\mathrm{Tp}=22.47 \pm 0.18^{\circ} \mathrm{C}\right.$ ) (KruskalWallis statistic: $67.67, p<0.0001$; Figure 6A), representing a difference of around $2^{\circ} \mathrm{C}$. The results showed that spider mites infected with Wolbachia preferred cooler temperatures than uninfected spider mites.

\section{DISCUSSION}

In this study, we found that thermal tolerance was lower for mites that were singly infected with either Wolbachia or Spiroplasma than for co-infected and uninfected mites (Supplementary Table 3). It is likely that spider mites singly infected with either Wolbachia or Spiroplasma have reduced thermal tolerance, but co-infection by the two endosymbionts confers a level of protection for their hosts. Host fitness costs associated with high-temperature stress appear to be magnified when spider mites are singly infected with either Wolbachia or Spiroplasma (Corbin et al., 2017). In contrast, the co-infecting endosymbionts may additively or synergistically confer greater tolerance in their host (Vautrin and Vavre, 2009) which may improve performance under stressful environmental conditions (Renoz et al., 2019).

While underlying mechanisms remain unclear, researchers have previously documented that endosymbionts are vulnerable to both high- and low-temperature stress and that temperaturechallenged insects with a disrupted symbiotic system often show elevated mortality and other defective phenotypes (Prado et al., 2010). Symbioses of Wolbachia and Spiroplasma with $T$. truncatus, like other heritable symbioses, appear vulnerable to temperature stress (Corbin et al., 2017). High temperatures can reduce endosymbiont density and even eliminate symbionts in various insects (Doremus et al., 2019). Wolbachia density can be reduced and lost within three generations when $T$. urticae is reared at $35^{\circ} \mathrm{C}$ (van Opijnen and Breeuwer, 1999). This also appears to be the case for $T$. truncatus, where Wolbachia is reduced over generations when the host is reared at $35^{\circ} \mathrm{C}$ but remains stable at 20 and $25^{\circ} \mathrm{C}$. Spiroplasma is sensitive to both warmer and cooler conditions in co-infected spider mites when compared to the normal rearing temperature of $25^{\circ} \mathrm{C}$. A similar phenomenon has been observed in D. nebulosa, where Spiroplasma was rapidly lost at $18^{\circ} \mathrm{C}$ 

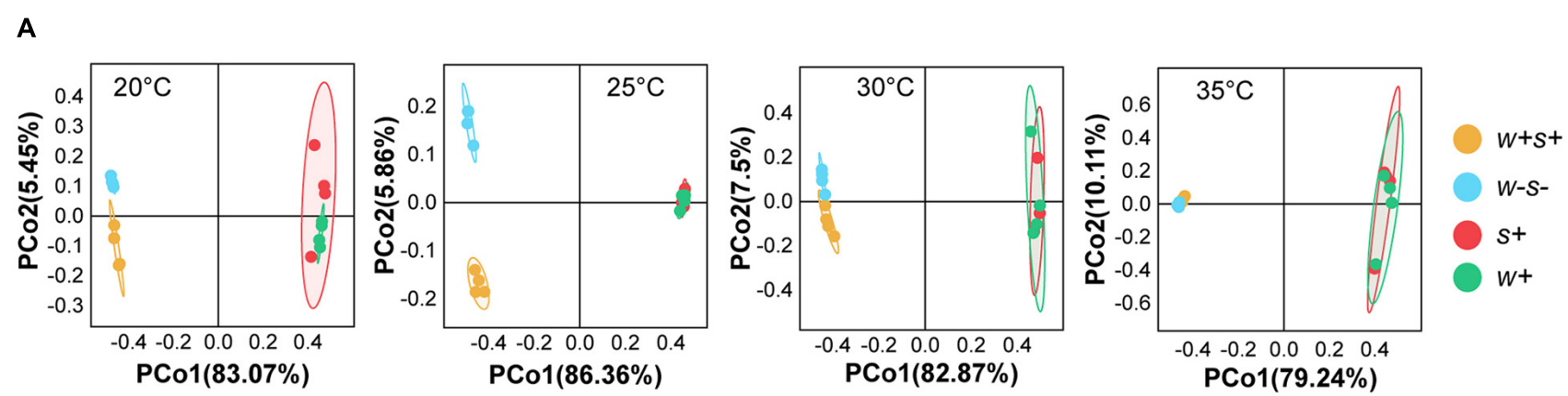

B
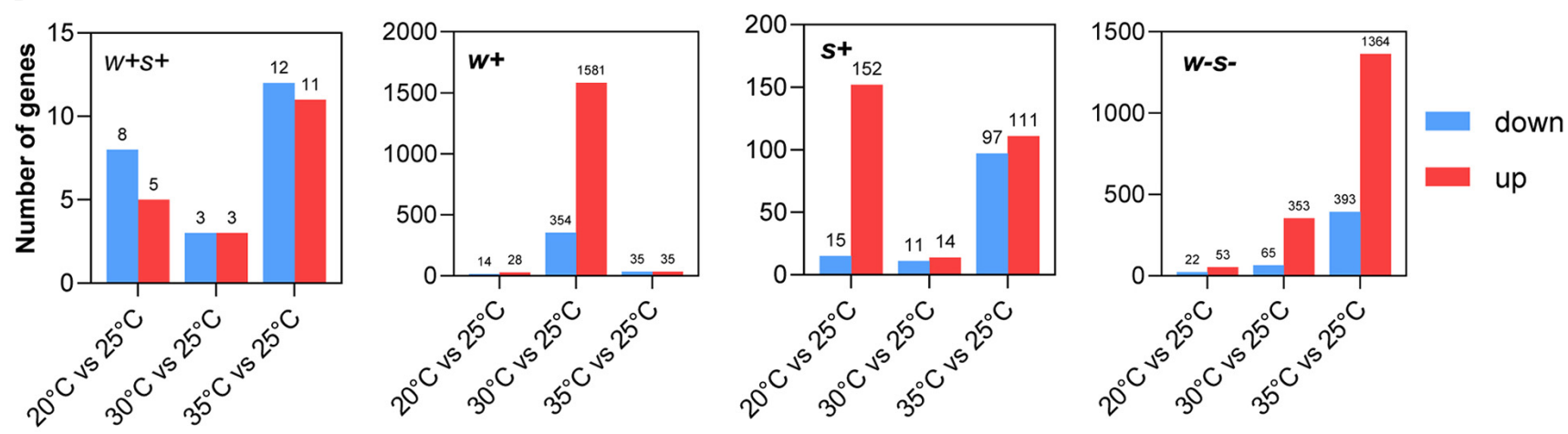

C

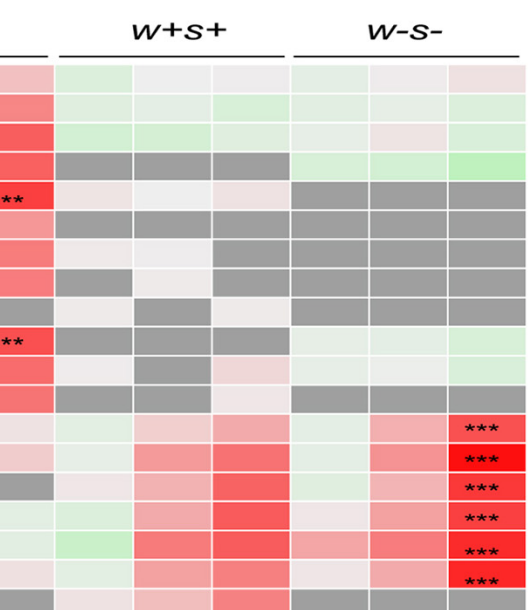

CL3734.Contig2 All (Hsp90) CL5629.Contig2_All (Hsp70) Unigene5373_All (Hsp20) Unigene5375_All (Hsp20) Unigene5380_All (Hsp20) Unigene5384 All (Hsp20) Unigene5382_All (Hsp20) Unigene5387_All (Hsp20) Unigene5386_All (Hsp20) Unigene5383_All ( $\mathrm{Hsp} 20)$ CL3754.Contig1_All (Hsp20) Unigene5378_All (Hsp20) CL212.Contig2_All (Hsp2O) CL3754.Contig2_All (Hsp20) Unigene5377_All (Hsp20) Unigene5385_All (Hsp20) CL212.Contig1_All (Hsp2O) CL212.Contig3_All (Hsp2O) Unigene7810_All (Hsp20)

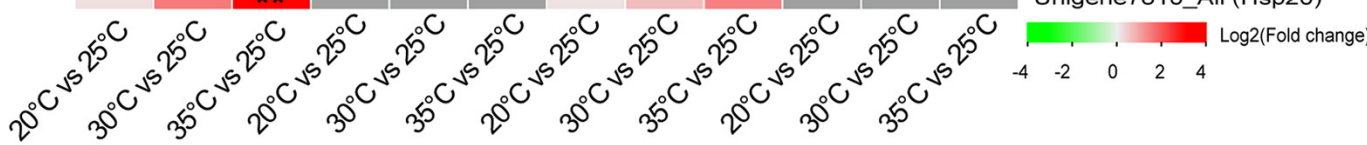

FIGURE 5 | Transcription response of four female adult spider mite strains following 6 h of different temperature exposures. (A) Principal components analysis (PCoA) of gene expression patterns of four spider mite strains under $20,25,30$, and $35^{\circ} \mathrm{C}$. (B) Number of differentially expressed genes (DEGs) with fold change $>2$ and an FDR-adjusted $p$-value of $<0.05$ for four spider mite strains under different temperatures. (C) Significantly differently expressed $H s p$ genes were analyzed by comparing each of 20,30 , and $35^{\circ} \mathrm{C}$ vs. the control $\left(25^{\circ} \mathrm{C}\right)$ based on a threshold of $>2$-fold change and an FDR-adjusted $p$-value of $<0.05$. The gray squares in the heat map indicate that gene expression was absent. Asterisks indicate statistically significant differences $\left({ }^{\star} p<0.05\right.$; ${ }^{* *} p<0.01$; and $\left.{ }^{* \star *} p<0.001\right)$.

and gradual loss occurred at $28^{\circ} \mathrm{C}$ (Anbutsu et al., 2008). Taken together, these results suggest that Wolbachia infections in mites differ from Spiroplasma in their response to lowand high-temperature stress, in that (i) Spiroplasma are more susceptible to high temperature than Wolbachia in either co-infected or singly infected strains, possibly due to Spiroplasma density being lower than Wolbachia density, and (ii) Wolbachia are more stable under high temperatures in doubly infected strains than in singly strains, whereas Spiroplasma seem more cold-sensitive in 

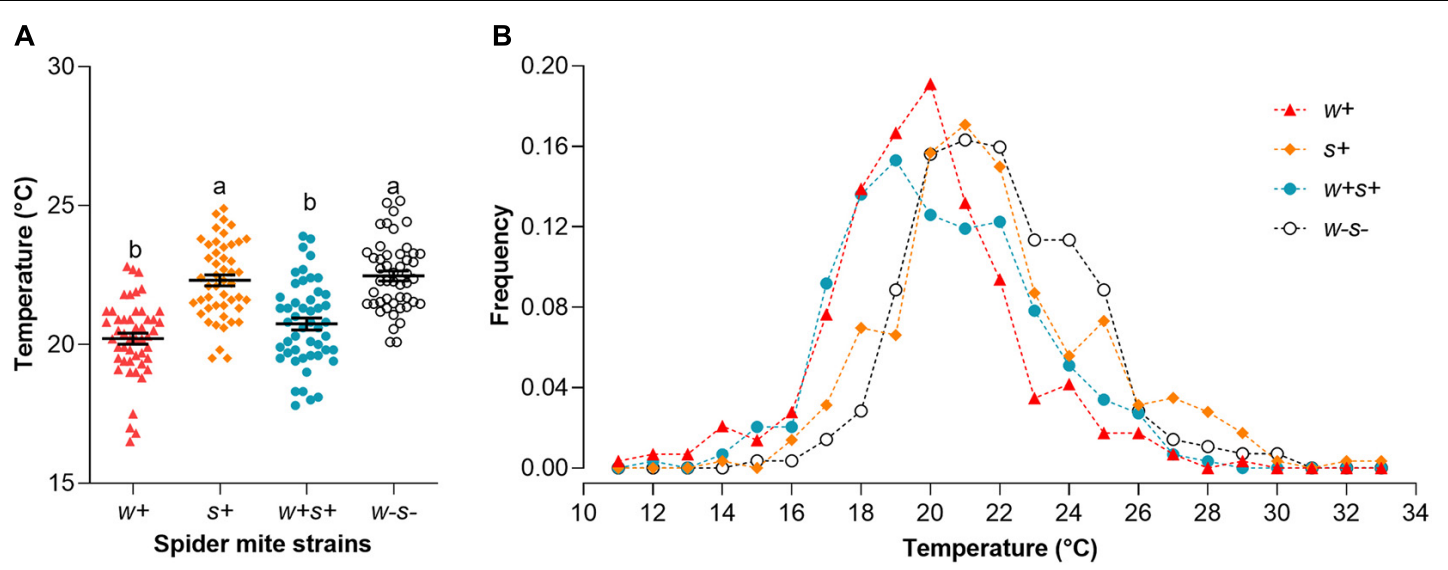

FIGURE 6| Thermal preference (Tp) of the four spider mite strains. (A) Average Tp of the four spider mite strains. Each dot on the graph indicates the mean of six individuals in each replicate. Long bar indicates the mean of all dots in the strain, and error bars indicate SE. Different letters indicate significant differences between four spider mite strains $(p<0.05)$. (B) Relative proportions of the four spider mite strains observed at a given temperature.

doubly infected strains than in singly infected strains. These patterns are consistent with the notion that temperature affects symbiont density and transmission in different ways, depending on the symbiont, host, and nature of the co-infection (Renoz et al., 2019).

Intriguingly, at both 30 and $20^{\circ} \mathrm{C}$, the frequency of Spiroplasma in co-infected strains decreased at the second generation and then fluctuated in later generations. When one of the co-infected endosymbionts causes CI, this helps to maintain not only the CI-causing endosymbiont but also any co-infecting endosymbiont via a hitchhiking effect (Engelstadter et al., 2004). Wolbachia induced incomplete CI in co-infected T. truncatus strains. Thus, the Spiroplasma infection which exhibited an improvement under high or low temperature in co-infected strains may be hitchhiking with co-infecting Wolbachia that induce CI. This also partly helps to explain why co-infections with multiple endosymbionts are common in natural populations of T. truncatus (Zhu et al., 2018).

Temperature-induced changes to endosymbionts densities are likely to have cascading effects. For example, high temperature decreased Wolbachia and Cardinium density and thus decreased CI levels in N. vitripennis and Euphorbia suzannae (Bordenstein and Bordenstein, 2011; Doremus et al., 2019). In contrast, Wolbachia quickly replicate in D. simulans (Clancy and Hoffmann, 1998) and Leptopilina heterotoma wasps (Mouton et al., 2006) at warmer temperature, and yet CI strength decreases. Thus, complex interactions between symbiont densities and temperature influence CI strength variation, and this process may involve other factors such as host behavior, development, host genetic variation (Shropshire et al., 2020), and especially activity of the CI enzyme CidB, which also varies with temperature (Beckmann et al., 2019). Here, we found that high and low temperatures may impact Wolbachia density and Wolbachia CI strength; however, there was no direct association between CI strength and Wolbachia density (Yang et al., 2020). In T. urticae, high temperature also reduced Wolbachia densities, but $\mathrm{CI}$ strength may associate with an increase in phage WO lytic activity ( $\mathrm{Lu}$ et al., 2012). This remains to be tested in T. truncatus.

In various heritable symbiotic systems, the thermal sensitivity of bacterial symbionts is likely to contribute to their ability to establish and persist in natural populations (Wernegreen, 2012). Wolbachia frequencies are high in spider mites across China and increase at localities with higher annual mean temperatures, while Spiroplasma is present at relatively low frequencies and patchily distributed (Zhu et al., 2018). The temperature conditions examined in this study are relevant to natural conditions experienced by mites in the field, although conditions there are variable, and it would be worth carrying out additional experiments under fluctuating temperatures (Ross et al., 2017).

We report for the first time that Wolbachia modifies $\mathrm{Tp}$ in spider mites, which might represent a behavioral accommodation to host-symbiont interactions (Truitt et al., 2019). Recently, Wolbachia strains in some Drosophila species were shown to prefer cooler temperatures (Arnold et al., 2019; Truitt et al., 2019; Hague et al., 2020), consistent with the current results. Differences in $\mathrm{Tp}$ between infected and uninfected flies could result from conflicting physiological requirements of Wolbachia and hosts (Hague et al., 2020), and this notion is worth further examination. We speculate that, as a consequence of trade-offs in thermal adaptation and balancing selection between the symbiont and the host, Wolbachiamediated host thermoregulatory behavior may affect mite thermoregulation, endosymbiont spread, and the maintenance of facultative symbioses.

The main molecular mechanisms of symbiont-mediated effects on host tolerance may involve endosymbiont-induced changes in stress-response genes (Brumin et al., 2011). Among these genes, Hsp genes such as Hsp70, Hsp90, and small heat shock protein ( $s H s p$ ) have been reported to play roles in hightemperature responses (Dahlgaard et al., 1998; Chen and Wagner, 2012). These proteins are molecular chaperones that promote 
correct refolding and prevent aggregation of denatured proteins in the face of a variety of stress factors, and thus may lead to host thermotolerance (King and MacRae, 2015). In the current study, the four spider mite strains differed in the expression changes of heat shock genes when exposed to different temperatures. The low number of DEGs in comparisons with the doubly infected strain suggests that co-infected strains are the least sensitive to different temperatures. In addition, Spiroplasma seems to interact with the relative expression of $H s p 70$ and $H s p 90$ at $20^{\circ} \mathrm{C}$. It is not yet clear if the differences in host gene expression are a consequence of responses to temperature, temperature effects on the endosymbionts, or some combination of these factors. The few DEGs in the doubly infected strain may reflect a relatively higher tolerance of this strain to high temperatures, so that host Hsps were not triggered to the same extent. Alternatively, symbiont encoded genes may affect host tolerance and directly affect host gene expression. For example, mutants of the symbiont Buchnera aphidicola with lower expression of the heat-shock gene $i b p A$ decrease heat tolerance of pea aphids (Zhang et al., 2019). Horizontal gene transfer (HGT) events between the bacteria and the host may also play a role and have recently been documented (Husnik et al., 2013; Husnik and McCutcheon, 2018). For instance, several genes horizontally transferred from diverse bacterial symbionts are known to be expressed in the host and can assist or enhance specific metabolic activities to promote host development (Manzano-Marín et al., 2020; Zhou et al., 2021), thus indirectly changing the thermal tolerance of the host. It is not clear whether HGT events between spider mites and endosymbionts produce similar effects.

\section{CONCLUSION}

In summary, we found that Wolbachia and Spiroplasma in spider mites may affect the fitness of spider mites at different temperatures. We also show that the nature of these effects depends on whether Wolbachia and Spiroplasma are present singly or together in the host. Endosymbionts may mediate host thermoregulatory behavior and influence $H s p$ expression under temperature stress. These results suggest that endosymbionts affect thermal responses of mites and raise issues about how ongoing climate change might influence the distribution of endosymbionts as well as their hosts.

\section{DATA AVAILABILITY STATEMENT}

All sequence data have been submitted to the NCBI Sequence Read Archive, BioProject accession number PRJNA717652.

\section{AUTHOR CONTRIBUTIONS}

$\mathrm{Y}-\mathrm{XZ}, \mathrm{AH}$, and $\mathrm{X}-\mathrm{YH}$ designed the research. Y-XZ, Z-RS, $\mathrm{Y}-\mathrm{YZ}$, and $\mathrm{X}-\mathrm{YH}$ performed the research. $\mathrm{Y}-\mathrm{XZ}, \mathrm{Z}-\mathrm{RS}, \mathrm{AH}$, and $\mathrm{X}-\mathrm{YH}$ wrote and edited the manuscript. All authors read and approved the manuscript.

\section{FUNDING}

This study was supported in part by the National Natural Science Foundation of China (32020103011, 31871976, and 31901888) and the Fundamental Research Funds for the Central Universities (KJQN202011).

\section{ACKNOWLEDGMENTS}

We would like to thank Shi-Mei Huo and Xue Xia of Nanjing Agricultural University (NJAU), China, for providing technical assistance with the experiments.

\section{SUPPLEMENTARY MATERIAL}

The Supplementary Material for this article can be found online at: https://www.frontiersin.org/articles/10.3389/fmicb. 2021.706321/full\#supplementary-material

Supplementary Figure 1 | Schematic representation of the study design. $w+s+, w+, s+$, and $w$-s- represent the spider mite strains infected with both Wolbachia and Spiroplasma, only Wolbachia, only Spiroplasma, and no endosymbionts, respectively. More than 282 mites from each strain were selected to test host Tp. The others were subjected to one of the four temperature treatments $\left(20,25,30\right.$, and $\left.35^{\circ} \mathrm{C}\right)$ for various time periods, depending on the experiment they were used for: (1) To assess survival rates at different temperatures, more than 116 female adults (2 days old) of each strain, grouped as six replicates of about 20 individuals, were exposed to each temperature treatment for a week with daily monitoring. (2) After $6 \mathrm{~h}$ of exposure to different temperatures, about 100 mites from each strain were collected for DNA extraction and transcriptome sequencing. (3) We collected mites exposed to different temperatures after $6,12,24,72$, and $120 \mathrm{~h}$ for testing densities of Wolbachia and Spiroplasma. (4) Each strain was reared for four generations under different temperature treatments. Twenty female adults were randomly selected from each combination of temperature and generation (F1, F2, F3, and F4) of these cultures, and used for PCR to detect the infection rates of Wolbachia and Spiroplasma. (5) In the crossing experiment, single females without Wolbachia (o: $s+$ or $w$-s-) in the teleiochrysalis stage were crossed with an adult virgin male (1 day old) infected with Wolbachia $\left(\sigma^{7}: w+s+\right.$ or $\left.w+\right)$ to produce four crosses. Mating and egg laying (up to 5 days) were carried out under one of the four temperature treatments, resulting in 16 total combinations, with 18-99 replicates each.

Supplementary Figure 2 | (A) Schematic of the thermal gradient apparatus. (B) Plots showing the length of the $500 \mathrm{~mm}$ aluminum gradient and where temperature was recorded with K-type thermocouples. Red dots indicate spider mite entry point. (C) Linearity of temperature change for the different surfaces as measured with K-type thermocouples.

Supplementary Figure $\mathbf{3}$ | Gene Ontology (GO) terms (A) and Kyoto Encyclopedia of Genes and Genomes (KEGG) terms (B) of genes identified from female Tetranychus truncatus.

Supplementary Figure 4 | Confirmation of gene expression by quantitative real-time PCR. The relative expression level of each gene was determined by qPCR (red) and was compared with the expression of the transcriptomic data (blue).

Supplementary Table 1 | Primers sequences. Sequences of DNA oligonucleotides used for assays described in this manuscript.

Supplementary Table 2 | Information on de novo assembled genes of female spider mites and annotations to different databases.

Supplementary Table $\mathbf{3}$ | Summarizes of endosymbiont infection pattern affects each of the host phenotypes. 


\section{REFERENCES}

Anbutsu, H., Goto, S., and Fukatsu, T. (2008). High and low temperatures differently affect infection density and vertical transmission of male-killing Spiroplasma symbionts in Drosophila hosts. Appl. Environ. Microb. 74, 60536059. doi: 10.1128/AEM.01503-08

Arnold, P. A., Levin, S. C., Stevanovic, A. L., and Johnson, K. N. (2019). Drosophila melanogaster infected with Wolbachia strain wMelcs prefer cooler temperatures. Ecol. Entomol. 44, 287-290. doi: 10.1111/een.12696

Beckmann, J. F., Sharma, G. D., Mendez, L., Chen, H., and Hochstrasser, M. (2019). The Wolbachia cytoplasmic incompatibility enzyme CidB targets nuclear import and protamine-histone exchange factors. Elife 8:e50026. doi: 10.7554/ eLife. 50026

Bordenstein, S. R., and Bordenstein, S. R. (2011). Temperature affects the tripartite interactions between bacteriophage WO, Wolbachia, and cytoplasmic incompatibility. PLoS One 6:e29106. doi: 10.1371/journal.pone.0029106

Brumin, M., Kontsedalov, S., and Ghanim, M. (2011). Rickettsia influences thermotolerance in the whitefly Bemisia tabaci B biotype. Insect Sci. 18, 57-66. doi: $10.1111 / \mathrm{j} .1744-7917.2010 .01396 . \mathrm{x}$

Burke, G., Fiehn, O., and Moran, N. (2010). Effects of facultative symbionts and heat stress on the metabolome of pea aphids. ISME J. 4, 242-252. doi: 10.1038/ ismej.2009.114

Chen, B., and Wagner, A. (2012). Hsp90 is important for fecundity, longevity, and buffering of cryptic deleterious variation in wild fly populations. BMC Evol. Biol. 12:25. doi: 10.1186/1471-2148-12-25

Clancy, D. J., and Hoffmann, A. A. (1998). Environmental effects on cytoplasmic incompatibility and bacterial load in Wolbachia-infected Drosophila simulans. Entomol. Exp. Appl. 86, 13-24. doi: 10.1046/j.1570-7458.1998.0 0261.x

Colinet, H., Sinclair, B. J., Vernon, P., and Renault, D. (2015). Insects in fluctuating thermal environments. Annu. Rev. Entomol. 60, 123-140. doi: 10. 1146/annurev-ento-010814-021017

Corbin, C., Heyworth, E. R., Ferrari, J., and Hurst, G. D. D. (2017). Heritable symbionts in a world of varying temperature. Heredity 118, 10-20. doi: 10.1038/ hdy. 2016.71

Dahlgaard, J., Loeschcke, V., Michalak, P., and Justesen, J. (1998). Induced thermotolerance and associated expression of the heat-shock protein Hsp70 in adult Drosophila melanogaster. Funct. Ecol. 12, 786-793. doi: 10.1046/j.13652435.1998.00246.x

Doremus, M. R., Kelly, S. E., and Hunter, M. S. (2019). Exposure to opposing temperature extremes causes comparable effects on Cardinium density but contrasting effects on Cardinium-induced cytoplasmic incompatibility. PLoS Pathog. 15:e1008022. doi: 10.1371/journal.ppat.1008022

Dunbar, H. E., Wilson, A. C., Ferguson, N. R., and Moran, N. A. (2007). Aphid thermal tolerance is governed by a point mutation in bacterial symbionts. PLoS Boil. 5:e96. doi: 10.1371/journal.pbio.0050096

Engelstadter, J., Telschow, A., and Hammerstein, P. (2004). Infection dynamics of different Wolbachia-types within one host population. J. Theor. Biol. 231, 345-355. doi: 10.1016/j.jtbi.2004.06.029

Gruntenko, N. E., Ilinsky, Y. Y., Adonyeva, N. V., Burdina, E. V., Bykov, R. A., Menshanov, P. N., et al. (2017). Various Wolbachia genotypes differently influence host Drosophila dopamine metabolism and survival under heat stress conditions. BMC Evol. Biol. 17:252. doi: 10.1186/s12862-017-1104-y

Hague, M. T. J., Caldwell, C. N., and Cooper, B. S. (2020). Pervasive effects of Wolbachia on host temperature preference. mBio 11, e1768-e1720. doi: 10 . 1128/mBio.01768-20

Heyworth, E. R., Smee, M. R., and Ferrari, J. (2020). Aphid facultative symbionts aid recovery of their obligate symbiont and their host after heat stress. Front. Ecol. Evol. 8:56. doi: 10.3389/fevo.2020.00056

Hoffmann, A. A., Turelli, M., and Simmons, G. M. (1986). Unidirectional incompatibility between populations of Drosophila simulans. Evolution 40, 692-701. doi: 10.1111/j.1558-5646.1986.tb00531.x

Husnik, F., and McCutcheon, J. P. (2018). Functional horizontal gene transfer from bacteria to eukaryotes. Nat. Rev. Microbiol. 16, 67-79. doi: 10.1038/nrmicro. 2017.137

Husnik, F., Nikoh, N., Koga, R., Ross, L., Duncan, R. P., Fujie, M., et al. (2013). Horizontal gene transfer from diverse bacteria to an insect genome enables a tripartite nested mealybug symbiosis. Cell 153, 1567-1578. doi: 10.1016/j.cell. 2013.05.040

Jin, P. Y., Tian, L., Chen, Lei, and Hong, X. Y. (2018). Spider mites of agricultural importance in China, with focus on species composition during the last decade (2008-2017). Syst. Appl. Acarol. 23, 2087-2098. doi: 10.11158/saa. 23.11.1

Kiers, E. T., Palmer, T. M., Ives, A. R., Bruno, J. F., and Bronstein, J. L. (2010). Mutualisms in a changing world: an evolutionary perspective. Ecol. Lett. 13, 1459-1474. doi: 10.1111/j.1461-0248.2010.01538.x

King, A. M., and MacRae, T. H. (2015). Insect heat shock proteins during stress and diapause. Annu. Rev. Entomol. 60, 59-75. doi: 10.1146/annurev-ento-011613162107

Lemoine, M. M., Engl, T., and Kaltenpoth, M. (2020). Microbial symbionts expanding or constraining abiotic niche space in insects. Curr. Opin. Insect Sci. 39, 14-20. doi: 10.1016/j.cois.2020.01.003

Livak, K. J., and Schmittgen, T. D. (2001). Analysis of relative gene expression data using real-time quantitative PCR and the $2^{-\Delta \Delta}$ CT method. Methods 25, 402-408. doi: 10.1006/meth.2001.1262

Love, M. I., Huber, W., and Anders, S. (2014). Moderated estimation of fold change and dispersion for RNA-seq data with DESeq2. Genome Biol. 15:550. doi: 10.1186/s13059-014-0550-8

Lu, M. H., Zhang, K. J., and Hong, X. Y. (2012). Tripartite associations among bacteriophage WO, Wolbachia, and host affected by temperature and age in Tetranychus urticae. Exp. Appl. Acarol. 58, 207-220. doi: 10.1007/s10493-0129578-1

Ma, C. S., Ma, G., and Pincebourde, S. (2021). Survive a warming climate: insect responses to extreme high temperatures. Annu. Rev. Entomol. 66, 163-184. doi: 10.1146/annurev-ento-041520-074454

Manzano-Marín, A., Coeur d'acier, A., Clamens, A. L., Orvain, C., Cruaud, C., Barbe, V., et al. (2020). Serial horizontal transfer of vitamin-biosynthetic genes enables the establishment of new nutritional symbionts in aphids' di-symbiotic systems. ISME J. 14, 259-273. doi: 10.1038/s41396-0190533-6

Montllor, C. B., Maxmen, A., and Purcell, A. H. (2002). Facultative bacterial endosymbionts benefit pea aphids Acyrthosiphon pisum under heat stress. Ecol. Entomol. 27, 189-195. doi: 10.1046/j.1365-2311.2002.00393.x

Mouton, L., Henri, H., Bouletreau, M., and Vavre, F. (2006). Effect of temperature on Wolbachia density and impact on cytoplasmic incompatibility. Parasitology 132, 49-56. doi: 10.1017/S0031182005008723

Prado, S. S., Hung, K. Y., Daugherty, M. P., and Almeida, R. P. (2010). Indirect effects of temperature on stink bug fitness, via maintenance of gut-associated symbionts. Appl. Environ. Microbiol. 76, 1261-1266. doi: 10.1128/AEM.02 034-09

Renoz, F., Pons, I., and Hance, T. (2019). Evolutionary responses of mutualistic insect-bacterial symbioses in a world of fluctuating temperatures. Curr. Opin. Insect Sci. 35, 20-26. doi: 10.1016/j.cois.2019.06.006

Ross, P. A., Axford, J. K., Yang, Q., Staunton, K. M., Ritchie, S. A., Richardson, K. M., et al. (2020). Heatwaves cause fluctuations in wMel Wolbachia densities and frequencies in Aedes aegypti. PLoS Negl. Trop. Dis. 14:e0007958. doi: 10. 1371/journal.pntd.0007958

Ross, P. A., Wiwatanaratanabutr, I., Axford, J. K., White, V. L., EndersbyHarshman, N. M., and Hoffmann, A. A. (2017). Wolbachia Infections in Aedes aegypti differ markedly in their response to cyclical heat stress. PLoS Pathog. 13:e1006006. doi: 10.1371/journal.ppat.1006006

Sgro, C. M., Terblanche, J. S., and Hoffmann, A. A. (2016). What can plasticity contribute to insect responses to climate change? Annu. Rev. Entomol. 61, 433-451. doi: 10.1146/annurev-ento-010715-023859

Shropshire, J. D., Leigh, B., and Bordenstein, S. R. (2020). Symbiont-mediated cytoplasmic incompatibility: What have we learned in 50 years? eLife 9:e61989. doi: 10.7554/eLife.61989

Truitt, A. M., Kapun, M., Kaur, R., and Miller, W. J. (2019). Wolbachia modifies thermal preference in Drosophila melanogaster. Environ. Microbiol. 21, 32593268. doi: 10.1111/1462-2920.14347

van Opijnen, T., and Breeuwer, J. A. J. (1999). High temperatures eliminate Wolbachia, a cytoplasmic incompatibility inducing endosymbiont, from the two-spotted spider mite. Exp. Appl. Acarol. 23, 871-881. doi: 10.1023/A: 1006363604916 
Vautrin, E., and Vavre, F. (2009). Interactions between vertically transmitted symbionts: cooperation or conflict? Trends Microbiol. 17, 95-99. doi: 10.1016/j. tim.2008.12.002

Wernegreen, J. J. (2012). Mutualism meltdown in insects: bacteria constrain thermal adaptation. Curr. Opin. Microbiol. 15, 255-262. doi: 10.1016/j.mib. 2012.02.001

Yang, K., Xie, K., Zhu, Y. X., Huo, S. M., Hoffmann, A. A., and Hong, X. Y. (2020). Wolbachia dominate Spiroplasma in the co-infected spider mite Tetranychus truncatus. Insect Mol. Biol. 29, 19-37. doi: 10.1111/imb. 12607

Zhang, B., Leonard, S. P., Li, Y., and Moran, N. A. (2019). Obligate bacterial endosymbionts limit thermal tolerance of insect host species. Proc. Natl. Acad. Sci. U. S. A. 116, 24712-24718. doi: 10.1073/pnas.1915307116

Zhou, X., Ling, X., Guo, H., Zhu-Salzman, K., Ge, F., and Sun, Y. (2021). Serratia symbiotica enhances fatty acid metabolism of pea aphid to promote host development. Int. J. Mol. Sci. 22:5951. doi: 10.3390/ijms22115951

Zhu, Y. X., Song, Y. L., Zhang, Y. K., Hoffmann, A. A., Zhou, J. C., Sun, J. T., et al. (2018). Incidence of facultative bacterial endosymbionts in spider mites associated with local environment and host plant. Appl. Environ. Microb. 84, e2546-e2517. doi: 10.1128/AEM. 02546-17

Zhu, Y. X., Song, Z. R., Huo, S. M., Yang, K., and Hong, X. Y. (2020). Variation in the microbiome of the spider mite Tetranychus truncatus with sex, instar and endosymbiont infection. FEMS Microbiol. Ecol. 96:fiaa004. doi: 10.1093/femsec/ fiaa004

Conflict of Interest: The authors declare that the research was conducted in the absence of any commercial or financial relationships that could be construed as a potential conflict of interest.

Copyright (c) 2021 Zhu, Song, Zhang, Hoffmann and Hong. This is an open-access article distributed under the terms of the Creative Commons Attribution License (CC BY). The use, distribution or reproduction in other forums is permitted, provided the original author(s) and the copyright owner(s) are credited and that the original publication in this journal is cited, in accordance with accepted academic practice. No use, distribution or reproduction is permitted which does not comply with these terms. 